\title{
Optimization of Belleville Spring by Inspecting Parameter Impacts
}

\author{
Alper Karaduman ${ }^{1}$, Mehmet Onur Genç 1* $^{*}$
}

${ }^{1}$ Valeo Automotive Systems, Bursa, Turkey

\begin{abstract}
Clutch disc transmits the torque by frictional forces from the engine to transmission. For this purpose, the clutch disc includes some components such a Belleville spring to provide vibration dampening. Belleville springs are used in order to perform the hysteresis function of disc assembly by creating frictional torque into the clutch system. The hysteresis function of the disc is needed to absorb oscillations and prevent noise issues. Belleville spring geometrical shape is critically important and it defines load characteristics. A load of Belleville spring mainly depends on dimensions of inner diameter, outer diameter, thickness, angle, and slot dimensions. This study investigates the single and multi-objective optimization methodology in the automotive clutch system design. This novel methodology presents the design development approach to powertrain system components.
\end{abstract}

Keywords: Frictional Torque, Clutch system, FEA, Optimization, Belleville spring

\author{
* Corresponding author \\ Mehmet Onur Genç \\ mehmet-onur.genc@valeo.com \\ Adress: Valeo Automotive Sys- \\ tems, Bursa, Turkey
}

Research Article

\author{
Manuscript \\ Received 22.10.2019 \\ Revised 23.12.2019 \\ Accepted 23.12.2019
}

Doi: 10.30939/ijastech..635732

\section{Introduction}

Clutch provides a temporary connection between engine and gearbox by transmitting engine torque and engine speed in addition to the clutch's comfort function. One of the comfort functions is to damp vibrations while transmitting torque and speed towards gearbox. The clutch compensates vibrations by means of radial and axial springs. The Bellevillespringwhich is inserted into Disc creates frictional force during transmission. The frictional force is obtained by compressing Belleville spring into disc assembly. The absorbing of vibration is a key according to the engine characteristic of the vehicle, and the required torque is calculated. In this study, the load calculation of Bellevillespringwas performed and optimized, then the result has been investigated.

Studies on Belleville spring are limited in the literature and found only a few studies about the FEA modeling of Belleville washer. The behavior of stress and deflection of Belleville spring compared, but no study on clutch Belleville spring has been found about parameter optimization to get the required load and stress values.

Performance comparison of Belleville spring under compressive load was investigated between simulation and experimental results. They investigated the stress and deflection of the Belleville spring between the experimental method and FEA method. They decreased deviation between results by changing element formation [1]. Friction clutch design was performed with dual

Belleville springs and friction plates. He decreased the number of required components by using Belleville spring as a friction plate [2]. The method was developed for predicting the deformation behavior of Belleville spring under axial loadings by using the minimum potential energy principle. Classical thin shell theory in a conical coordinate system was used to formulate Belleville springs by strain energy and work. Their method brings more accurate results between experiments and the finite element method than almen and lazslo equation [3]. Composite materials were applied on Belleville springs instead of steel and composite materials provide identical load and deflection characteristics with steel [4].

Less interest has been seen to Belleville spring as clutch elements, no study has been found about shape optimization of Belleville spring to have target curve under stress constraints.

In this study, the shape optimization of Belleville spring has been done. Load and stress results were obtained by the finite element method. The design of the experiment was applied to the Latin hypercube sampling method. An evolutionary 
optimization algorithm was selected for shape optimization into the Optislang software.

\section{Material and Method}

Belleville spring which is compressed in the clutch has a conical shape, so it acts as spring during the engagement. Belleville spring is compressed into disc assembly as shown in Figure 1.

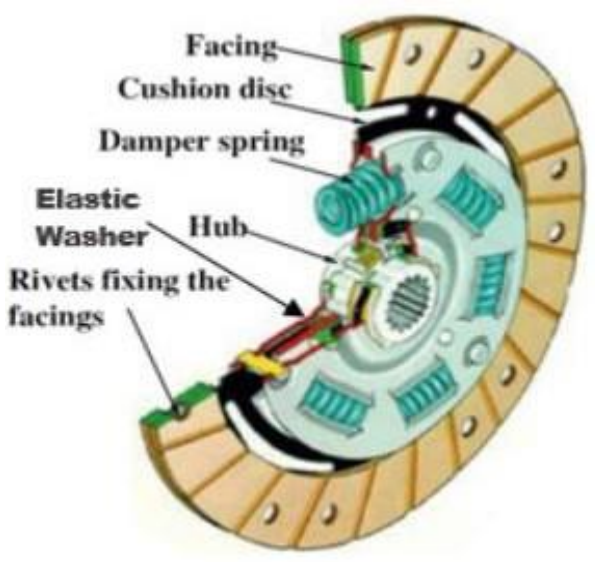

Fig. 1. Clutch disc mechanism

Load and stress values are taken by applying compressive displacement between two plates. The load-deflection curve is sinusoidal characteristic as seen in Figure 2. Load value needs to be between maximum and minimum limit curves while stress value is below the limit.

The finite element model was defined and conducted as nonlinear by using Ansys workbench so as to determine the load and the stress of the Belleville washer. Optimization was performed via the Optislang software. The solid geometry of the Belleville spring is shown in Figure 3.

The Belleville spring is compressed between upper and lower plates by applying displacement in the axial direction. Both sides of the Belleville spring includes cyclic boundary conditions. Displacement was applied from the plate to get force and stress values.

Belleville spring geometry is cyclic symmetric so $1 / 12$ model is considered in finite element model. Solid elements is applied. Lineer Belleville material is used. Finite element model is shown in Figure 4.

Belleville spring is compressed by giving axial displacement from the upper plate which is free on axial translation while the lower plate is fixed. Frictionless contacts were employed between Belleville spring and rigid plates.

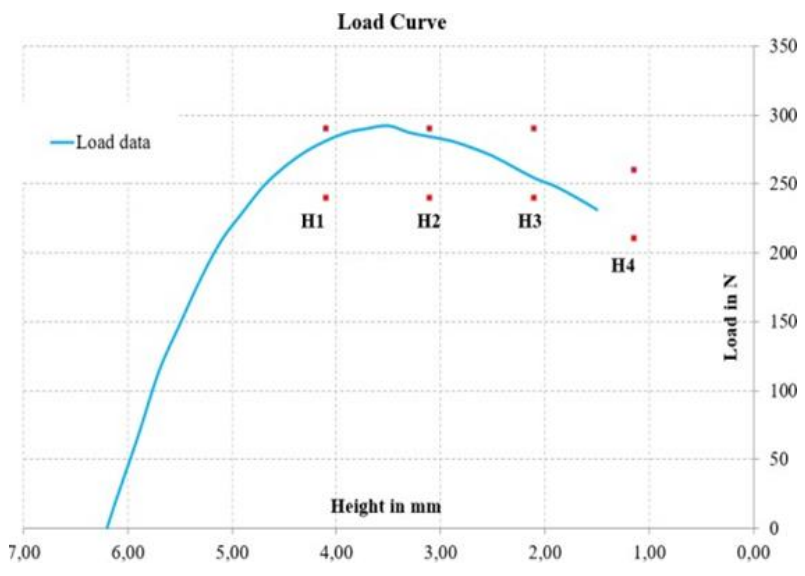

Fig. 2. A typical load curve of Bellevillespringand its limits

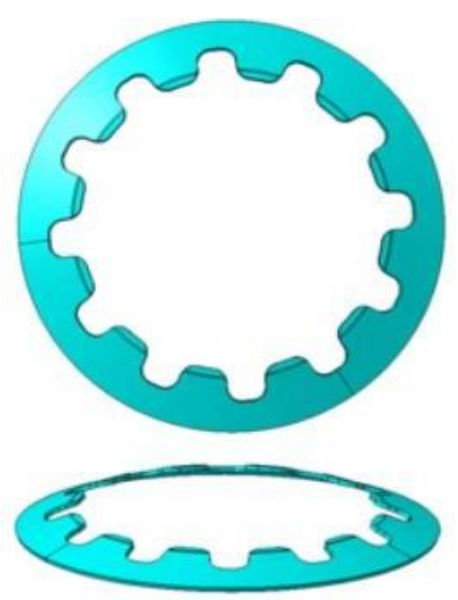

Fig. 3. Belleville spring solid geometry

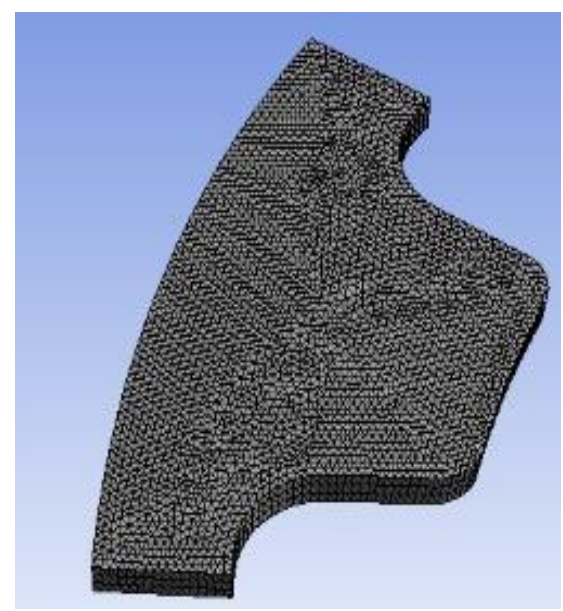

Fig. 4. 1/12 cyclic symmetry finite element model 


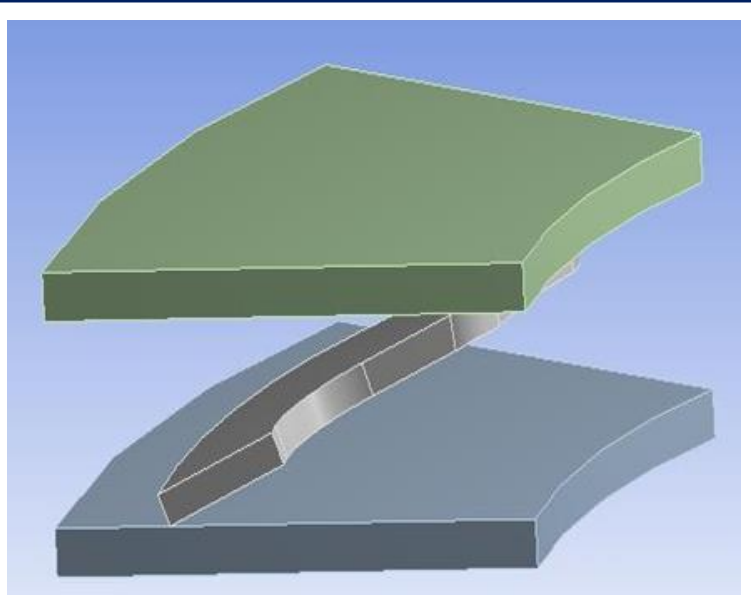

Fig. 5. Rigid plates
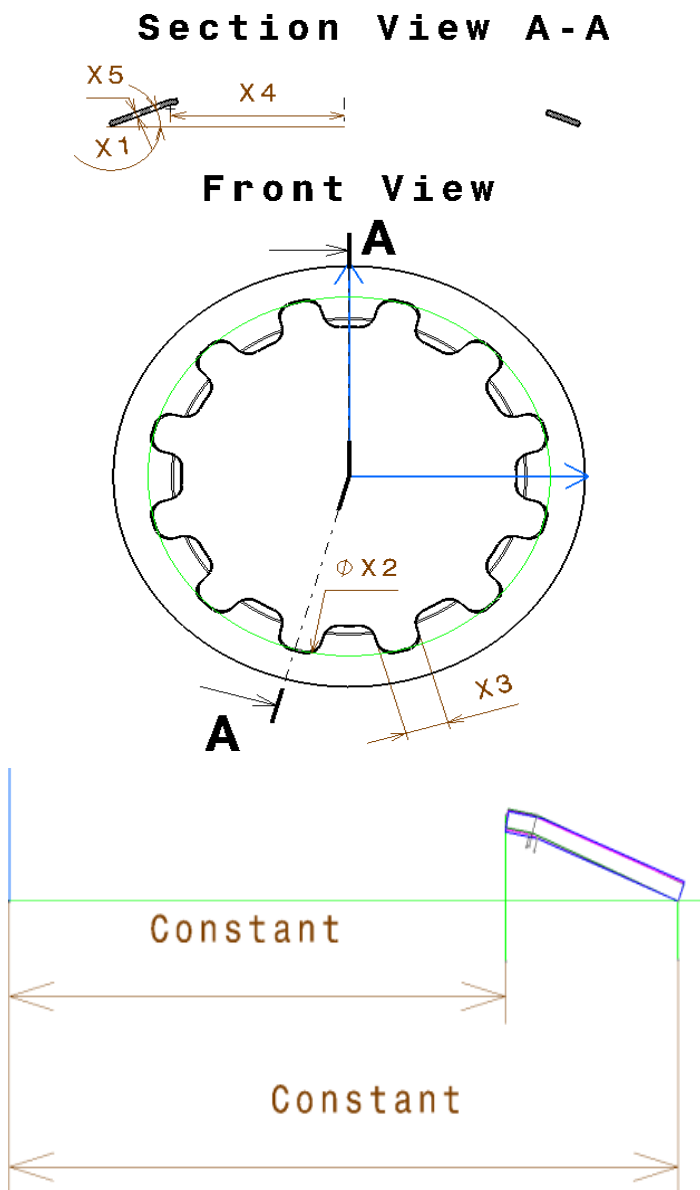

Fig. 6. Design parameters of Belleville spring

\section{Design of Experiment and Shape Optimization}

The calculation time is so important for structural analysis and the disadvantage of optimization studies. Meta-models are used to catch the relationship between variables and responses into the structural analysis.
This process is done to find the global respond of the objective function and constraint function. In addition to the aim of decreasing computation time and increasing sensitivity of target results, then the mathematical model was created by terms of collected data into the design of experiments so as to find optimum design parameters subjected to objective and constraint functions.

In this study, five design variables are considered while inner and outer diameters of Belleville spring are fixed. The design variables are as seen in Figure 6.

The advance Latin hypercube sampling method was applied in this study because it reduces sample size and unwanted input correlation.

There are five geometrical parameters to be selected to find parameter impacts under given same displacement. Furthermore, the maximum stress has to be below the allowable limit of its material. The design of Belleville spring is to find optimum parameters for its five geometric variables so as to meet the load requirements.

Table 1. Design table for a definition of parameter impacts

\begin{tabular}{|l|l|l|l|l|l|l|c|}
\hline & \multicolumn{5}{|c|}{ Parameters } & \multicolumn{2}{c|}{ Output } \\
\hline Run & $\mathbf{1}$ & $\mathbf{2}$ & $\mathbf{3}$ & $\mathbf{4}$ & $\mathbf{5}$ & $\begin{array}{l}\text { Y2 } \\
\text { S1 }\end{array}$ & $\begin{array}{l}\text { Y1 } \\
\text { Load }\end{array}$ \\
\hline $\mathbf{1}$ & 22.23 & 8.26 & 0.7 & 21.9 & 5 & 1545 & 31.8 \\
\hline $\mathbf{2}$ & 21.55 & 8.1 & 4.7 & 22.76 & 0.6 & 1141 & 25.1 \\
\hline $\mathbf{3}$ & 20.61 & 9.3 & 5.3 & 23 & 0.7 & 897 & 11.7 \\
\hline $\mathbf{4}$ & 20.78 & 9.6 & 4.5 & 22.6 & 0.7 & 1118 & 8.7 \\
\hline $\mathbf{5}$ & 21.01 & 8.6 & 5 & 22.9 & 0.7 & 979 & 17.6 \\
\hline $\mathbf{6}$ & 22.01 & 9.4 & 5 & 22.6 & 0.6 & 1088 & 9.8 \\
\hline $\mathbf{7}$ & 22.88 & 8.5 & 5.1 & 22.5 & 0.6 & 1343 & 23.9 \\
\hline $\mathbf{8}$ & 20.75 & 8.4 & 5.2 & 22.4 & 0.8 & 1131 & 23.9 \\
\hline $\mathbf{9}$ & 21.78 & 8.8 & 5.3 & 22.94 & 0.66 & 961 & 13.9 \\
\hline $\mathbf{1 0}$ & 20.91 & 8.7 & 5 & 22.1 & 0.63 & 973 & 13.5 \\
\hline $\mathbf{1 1}$ & 21.75 & 7.3 & 5.2 & 22.7 & 0.64 & 1071 & 34.9 \\
\hline $\mathbf{1 2}$ & 21.05 & 9.6 & 4.7 & 22.1 & 0.61 & 1048 & 7.1 \\
\hline $\mathbf{1 3}$ & 20.85 & 6.82 & 4.9 & 22.8 & 0.67 & 997 & 41.5 \\
\hline $\mathbf{1 4}$ & 21.65 & 8.3 & 4.8 & 22.9 & 0.62 & 958 & 17.9 \\
\hline, &.. &.. &.. &.. &.. &.. &.. \\
\hline,$\cdots$ & $\cdots$ & $\ldots$ & $\ldots$ & $\ldots$ & $\ldots$ & $\ldots$ & $\ldots$ \\
\hline $\mathbf{7 0}$ & 21.71 & 8.22 & 5.02 & 22.22 & 0.63 & 1161 & 22.25 \\
\hline & & & & & & & \\
\hline
\end{tabular}


Moreover, Optimum angle and forming diameter are to find for two of optimized parameters of Belleville spring since the angle and forming diameter defines the free height of Belleville spring which is so crucial parameter for load and stress optimization. Design of explorer, parameter impacts detection and optimization are conducted in OPTISLANG in ANSYS.

The objective function is to be minimized as follows. Objective function : min stress

*Min $[\mathrm{f}(\mathrm{x} 1, \mathrm{x} 2, \mathrm{x} 3, \mathrm{x} 4, \mathrm{x} 5)$ Stress] at $2 \mathrm{~mm}$ displacement

Constraint function : required load limits at $2 \mathrm{~mm}$ displacement.

$* 22.5<[\mathrm{f}(\mathrm{x} 1, \mathrm{x} 2, \mathrm{x} 3, \mathrm{x} 4, \mathrm{x} 5)$ Load $]<26.7$ for $1 / 12$ portion.

Each geometric parameter has its lower and upper bounds (Figure 7).

\begin{tabular}{|l|l|l|l|lllll|}
\hline \multicolumn{1}{|c}{ Name } & Parameter type & Reference value & Constant & Resolution & Range & Range plot \\
\hline Angle_ew & Optimization & 22.535 & $\square$ & Continuous & 20.5 & 23 & \\
\hline Forming_Diam & Optimization & 22.5 & $\square$ & Continuous & 22.1 & 23 & \\
\hline 3 Thickness & Optimization & 0.7 & $\square$ & Continuous & 0.6 & 0.8 & \\
\hline 4 Belleville_Dimension & Optimization & 8.26 & $\square$ & Continuous & 6.5 & 9.7 & \\
\hline 5 Belleville_width & Optimization & 5 & $\square$ & Continuous & 4.5 & 5.5 & \\
\hline
\end{tabular}

Fig. 7. Lower and upper bounds for optimization

Fitting of design of experiments is measured with coefficient of determination, $\mathrm{r} 2$, calculated as $\min 0.90$ for response functions.

\subsection{Parameter Analysis}

Results show that some of the parameters do not have any important impact on the objective function of Y2 "stress". The most important parameters are for Y2 "stress" X1, X2, $\mathrm{X} 3$ as seen in Figure 8. In addition, most important are for Y1 "Load", X3 and X4 as seen in Figure 8. Variable X5 does not have any impact on load characteristic of Belleville spring.
Table 2. Design table for load and stress optimization

\begin{tabular}{|l|c|l|c|l|c|c|}
\hline & \multicolumn{2}{|c|}{ Parameters } & \multicolumn{3}{c|}{ Output } \\
\hline Run & $\mathbf{2}$ & $\mathbf{3}$ & $\mathbf{5}$ & Y1 & Y2 & Y4 \\
\hline $\mathbf{1}$ & & & & & & \\
\hline $\mathbf{2}$ & 8.26 & 5.00 & 0.70 & 27.46 & 1559 & 381 \\
\hline $\mathbf{3}$ & 7.67 & 5.31 & 0.65 & 28.17 & 1546 & 509 \\
\hline $\mathbf{4}$ & 6.96 & 5.49 & 0.63 & 33.90 & 1545 & 722 \\
\hline $\mathbf{5}$ & 9.10 & 4.91 & 0.73 & 22.37 & 1573 & 233 \\
\hline $\mathbf{6}$ & 8.10 & 4.67 & 0.66 & 25.71 & 1549 & 370 \\
\hline $\mathbf{7}$ & 7.39 & 4.98 & 0.62 & 29.07 & 1548 & 551 \\
\hline $\mathbf{8}$ & 7.89 & 4.64 & 0.63 & 25.35 & 1548 & 398 \\
\hline $\mathbf{9}$ & 7.25 & 5.42 & 0.75 & 43.95 & 1552 & 735 \\
\hline $\mathbf{1 0}$ & 9.59 & 5.40 & 0.76 & 19.50 & 1586 & 185 \\
\hline $\mathbf{1 1}$ & 6.75 & 5.27 & 0.79 & 55.40 & 1553 & 893 \\
\hline $\mathbf{1 2}$ & 8.67 & 5.09 & 0.78 & 30.58 & 1569 & 344 \\
\hline $\mathbf{1 3}$ & 8.53 & 4.84 & 0.68 & 23.59 & 1561 & 303 \\
\hline $\mathbf{1 4}$ & 7.96 & 5.16 & 0.64 & 25.13 & 1557 & 416 \\
\hline $\mathbf{1 5}$ & 6.61 & 4.69 & 0.61 & 36.43 & 1547 & 789 \\
\hline $\mathbf{1 6}$ & 7.32 & 4.87 & 0.62 & 28.98 & 1545 & 559 \\
\hline $\mathbf{1 7}$ & 6.82 & 4.60 & 0.80 & 55.01 & 1567 & 797 \\
\hline $\mathbf{4 5}$ & 8.88 & 5.33 & 0.74 & 24.19 & 1572 & 289 \\
\hline & & &.. &.. &.. &.. \\
\hline
\end{tabular}

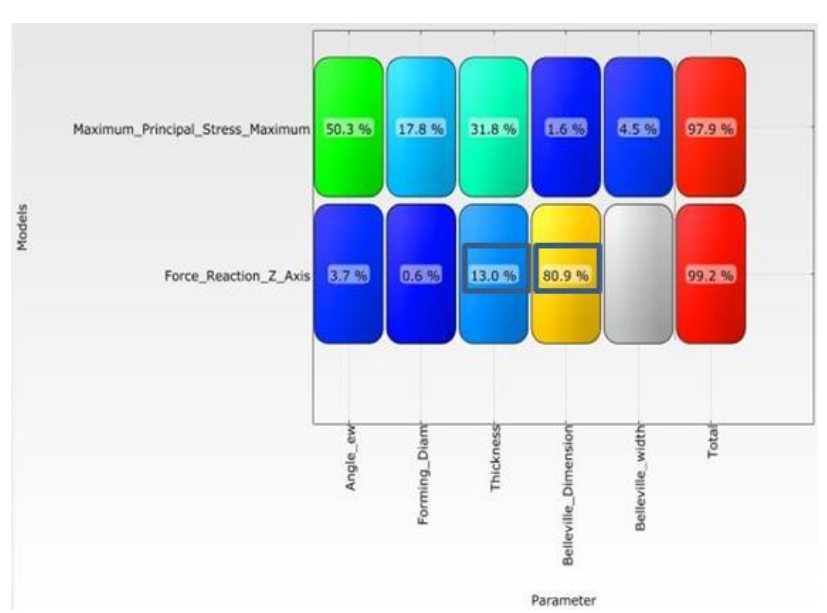

Fig. 8. Relationship between inputs and outputs 
As a result of the design of experiments created with advanced Latin hypercube sampling helps the creation of design space with a smaller number of samples and reducing unrealistic relationships between design variables. Sensitivity analysis into the Optislang software measures the coefficient of the importance of design variables by response functions. The relationship between design variables and responses is as seen in figures which are illustrated between Figure 9 and Figure 18.

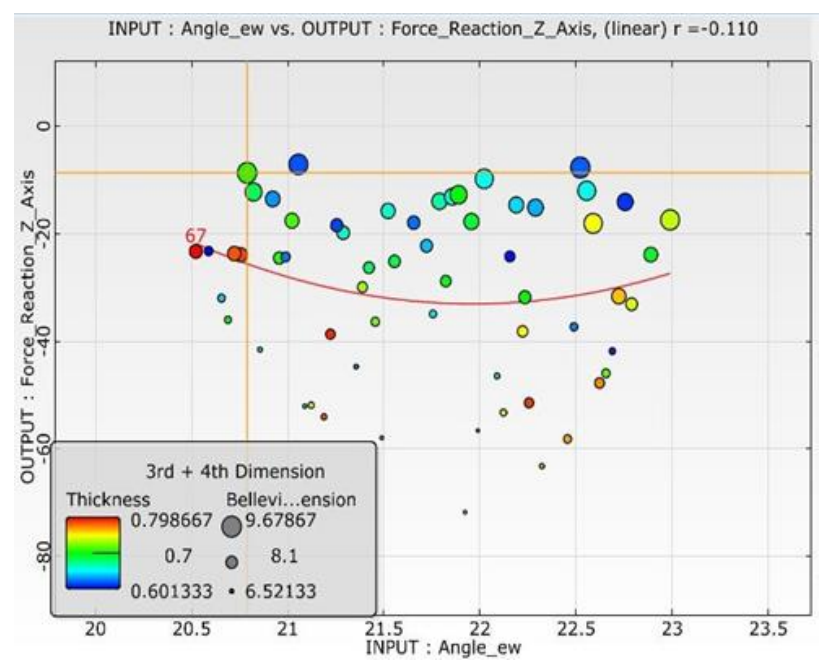

Fig. 9. Relationship between angle, thickness, Belleville diameter and load (Inner and outer diameters fixed)

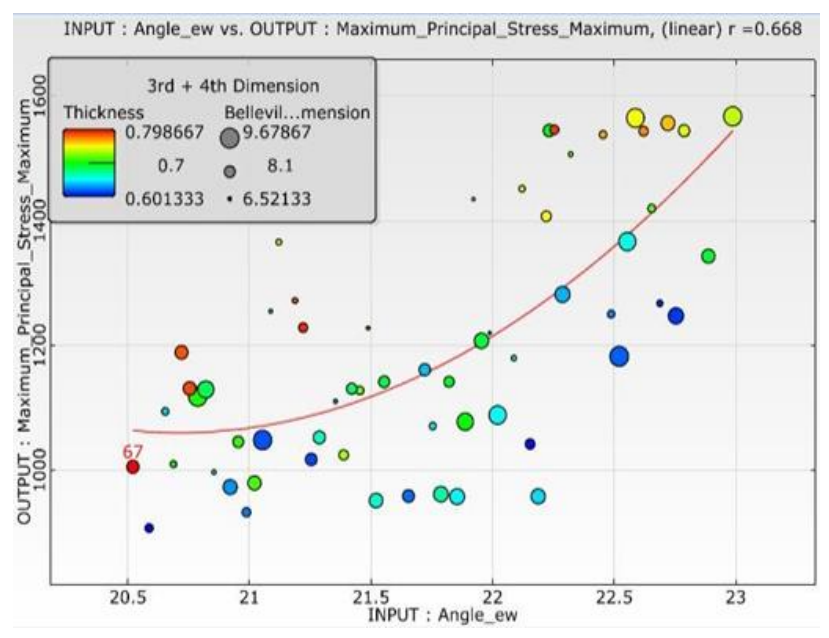

Fig. 10. Relationship between angle, thickness, Belleville diameter and stress (Inner and outer diameters fixed)

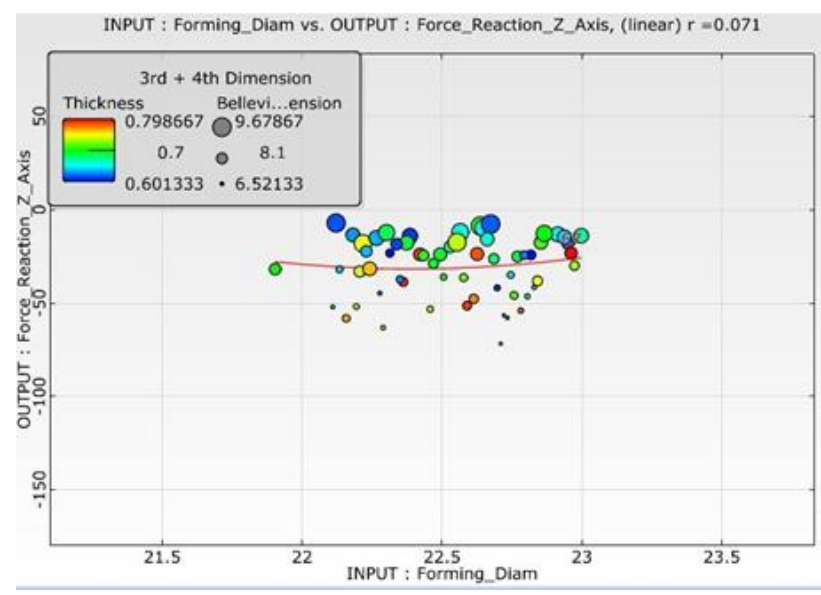

Fig. 11. Relationship between forming diam., thickness, Belleville diameter and load

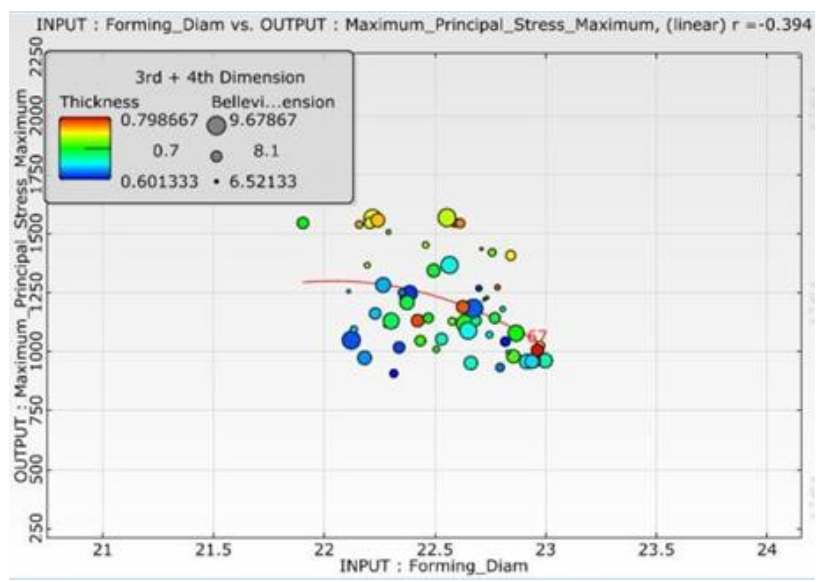

Fig. 12. Relationship between forming diam., thickness, Belleville diameter and stress

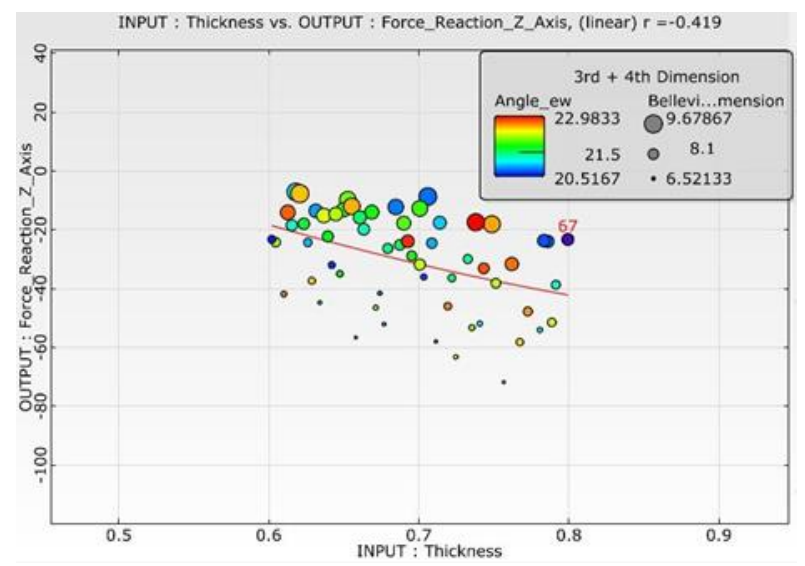

Fig. 13. Relationship between thickness, angle, belleville diameter and load 


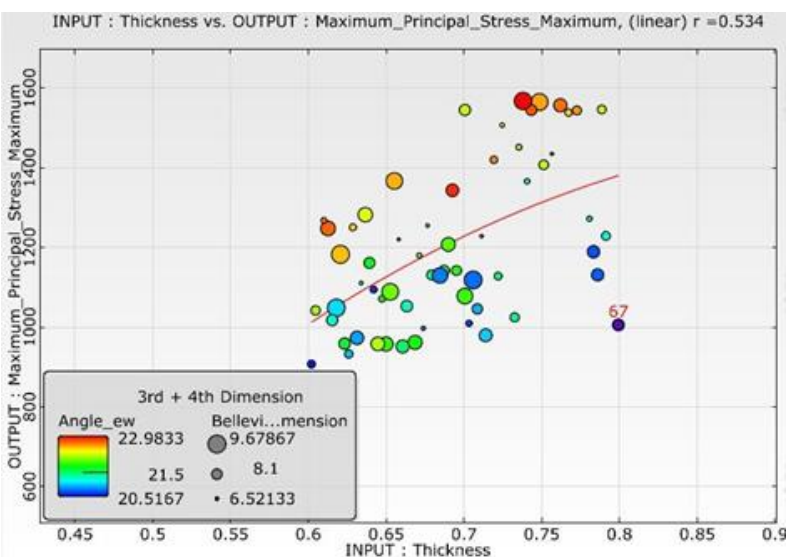

Fig. 14. Relationship between thickness, angle, belleville diameter and stress

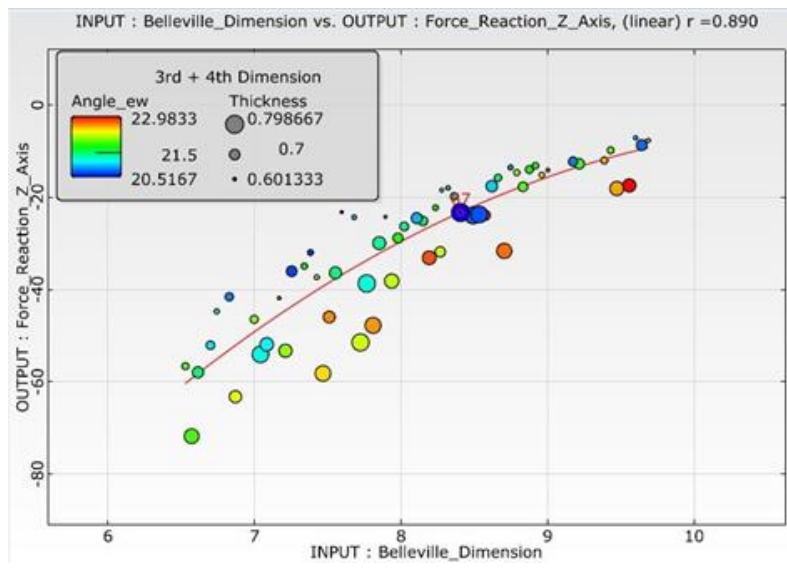

Fig. 15. Relationship between belleville diameter, thickness, angle and load

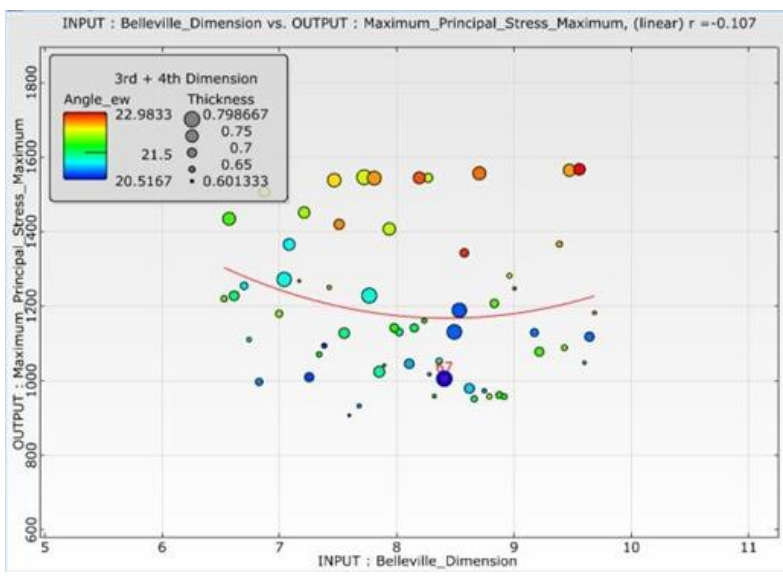

Fig. 16. Relationship between Belleville diameter, thickness, angle and stress

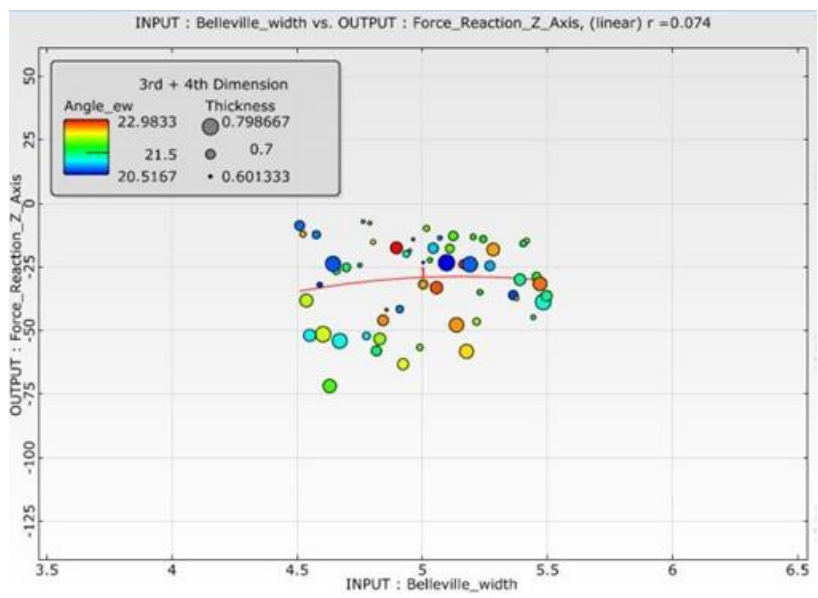

Fig. 17. Relationship between Belleville width, thickness, angle and load

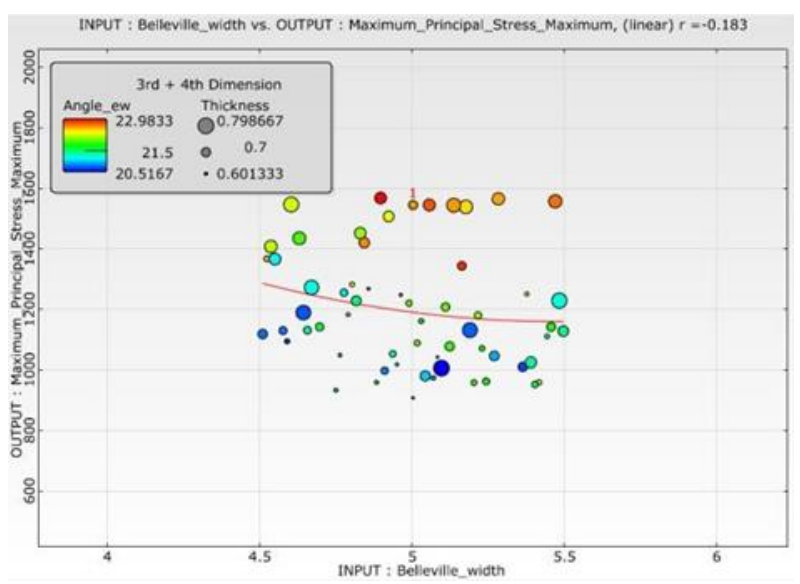

Fig. 18. Relationship between Belleville width, thickness, angle and stress

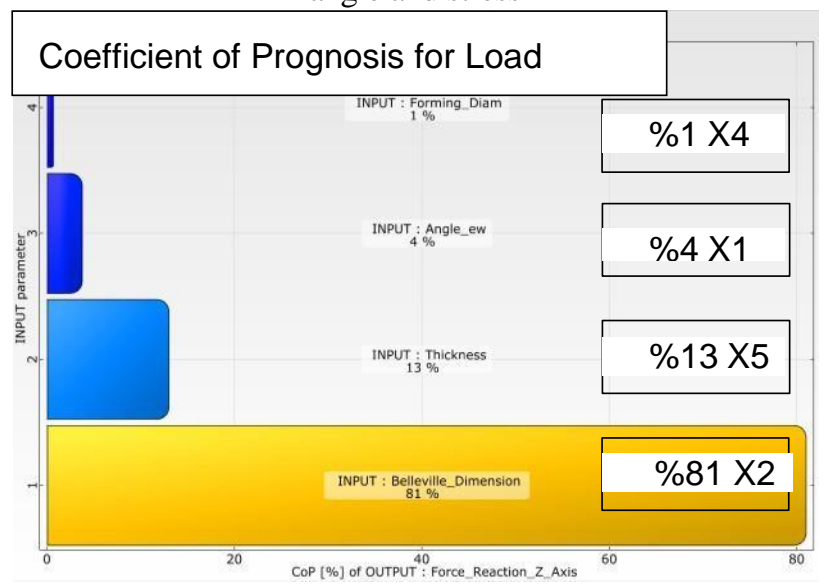

Fig. 19. Parameter impacts for Force 


\subsection{Optimization}

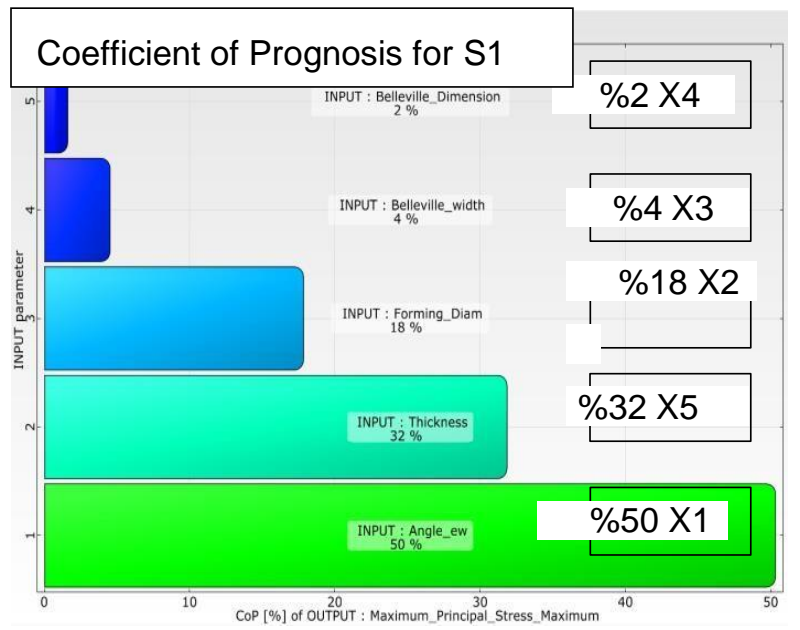

Fig. 20. Parameter impacts for Stress

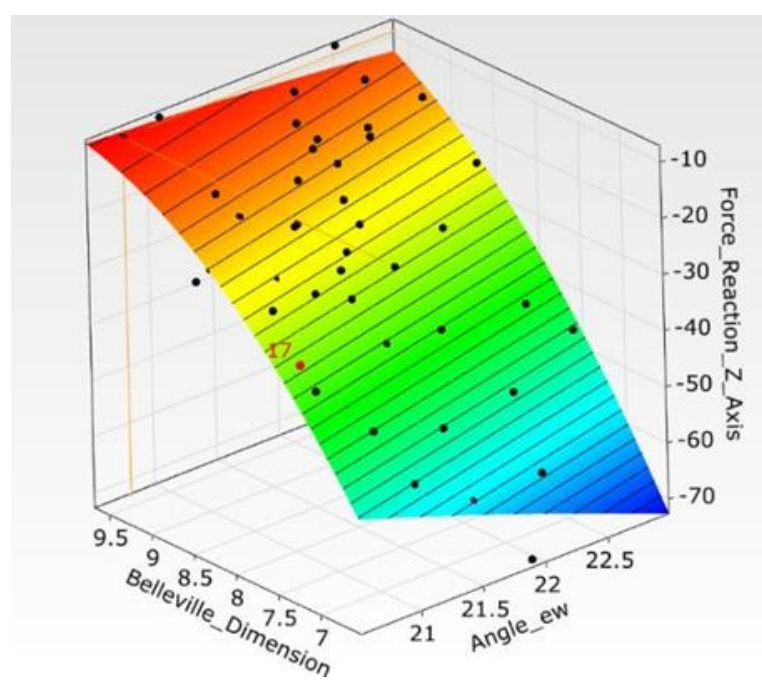

Fig. 21. Response surface by angle and Belleville d. for Force

Table 3. Optimum angle and forming diameter definition

\begin{tabular}{|c||l|l|l|l|l|l|}
\hline $\begin{array}{c}\text { Variables } \\
(\mathbf{m m})\end{array}$ & $\mathrm{X} 2$ & $\mathrm{X} 3$ & $\mathrm{X} 4$ & $\mathrm{X} 5$ & $\begin{array}{l}\text { Y1 } \\
(\mathrm{N})\end{array}$ & $\begin{array}{c}\text { Y2 } \\
(\mathrm{MPa})\end{array}$ \\
\hline $\begin{array}{c}\text { Opt. } \\
\text { NLPQL }\end{array}$ & 7,53 & 5,1 & $\begin{array}{c}22, \\
45\end{array}$ & 0,6 & 22,5 & 900 \\
\cline { 5 - 7 } & & & & Min & Min \\
\hline
\end{tabular}

After definition of optimum angle and forming diameter under same axial compression, optimization phase was initiated to define $\mathrm{x} 2, \mathrm{x} 3$ and $\mathrm{x} 5$ parameters in order to complete load and stress optimization. Most important parameters were found as X2 (belleville diameter) for Y4 (stiffness), as $\mathrm{X} 2$ (belleville diameter) and X3(belleville width) for $\mathrm{Y} 2$ (principal stress), as $\mathrm{X} 2$ (belleville diameter) and $\mathrm{X} 5$ (thickness) for Y1 (Load).

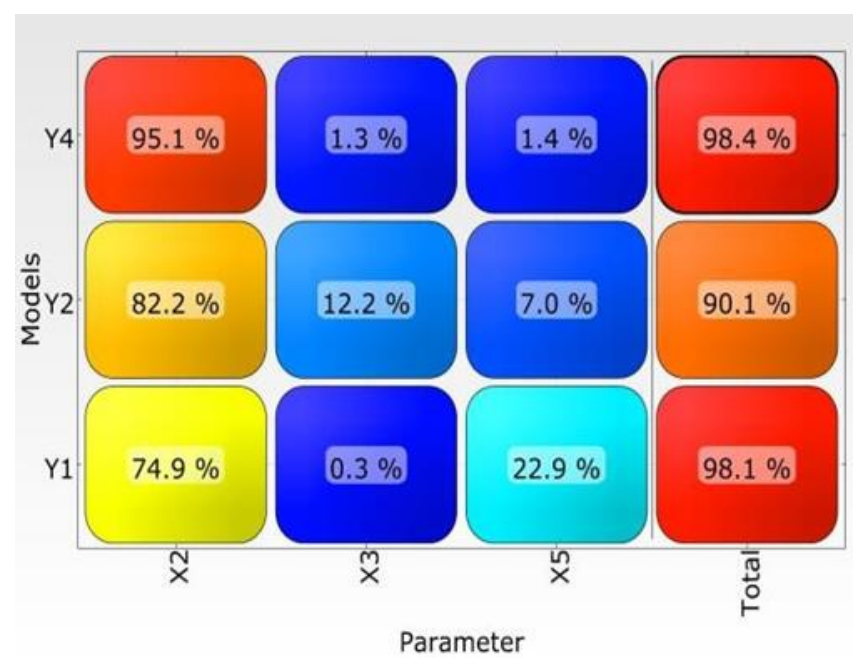

Fig. 22. Relationship between inputs and outputs

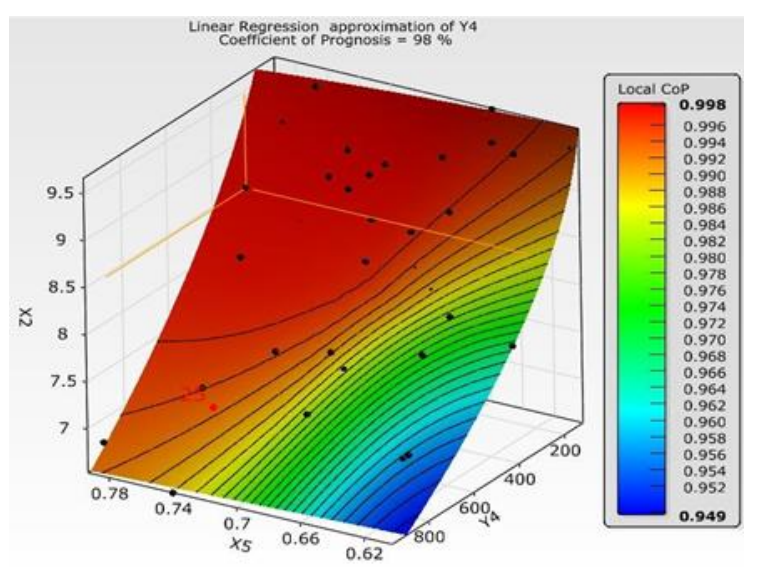

Fig. 23. Response surface by X2 "Belleville diam." and X5 "thickness" for Y4 "stiffness" 


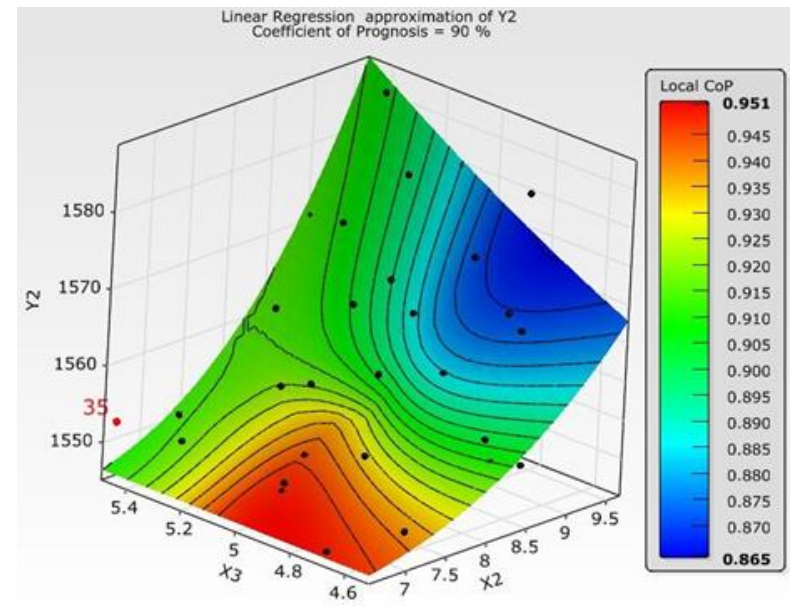

Fig. 24. Response surface by $\mathrm{x} 2$ "Belleville diam." and $\mathrm{x} 3$ "Belleville width" for Y2 "Principal Stress"

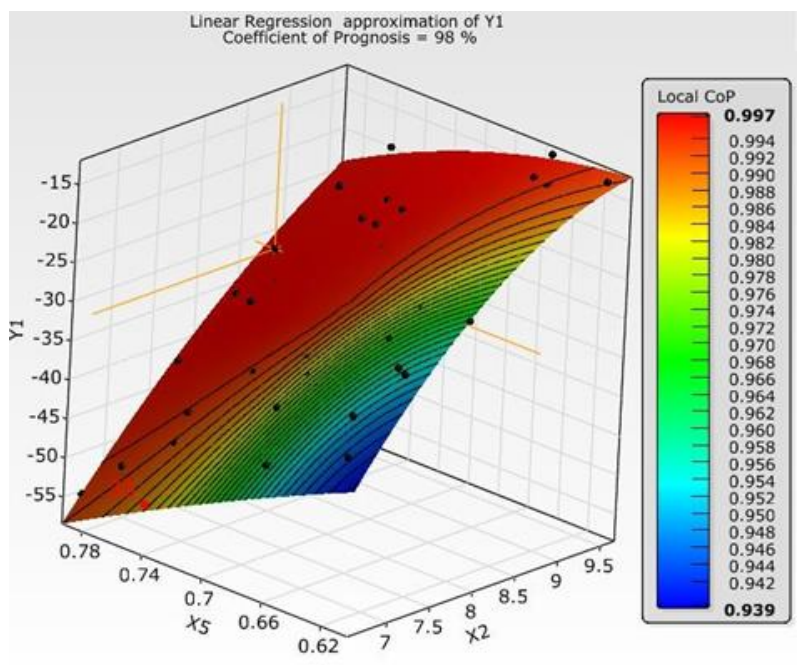

Fig. 25. Response surface by $x 2$ "belleville diam." and x 5 "thickness" for Y1 "Load"

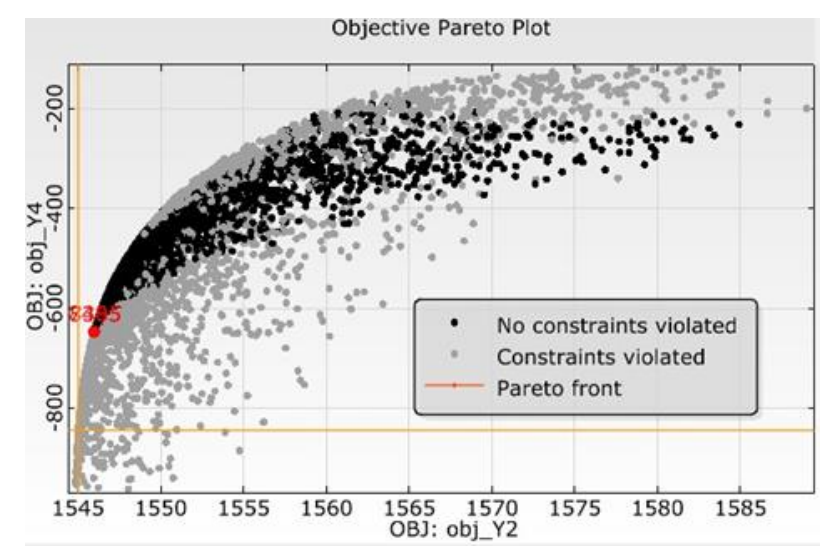

Fig. 26. Result from optimization

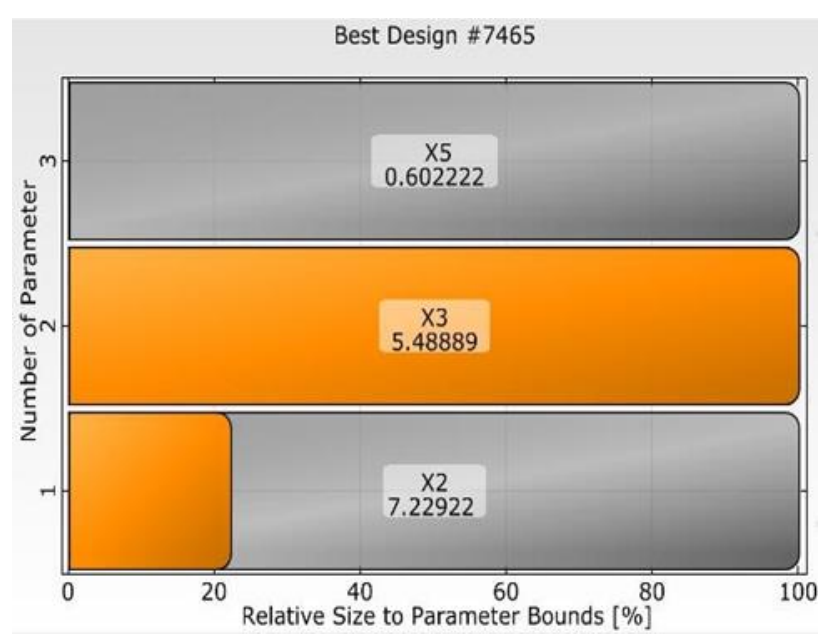

Fig. 27. Optimization parameter

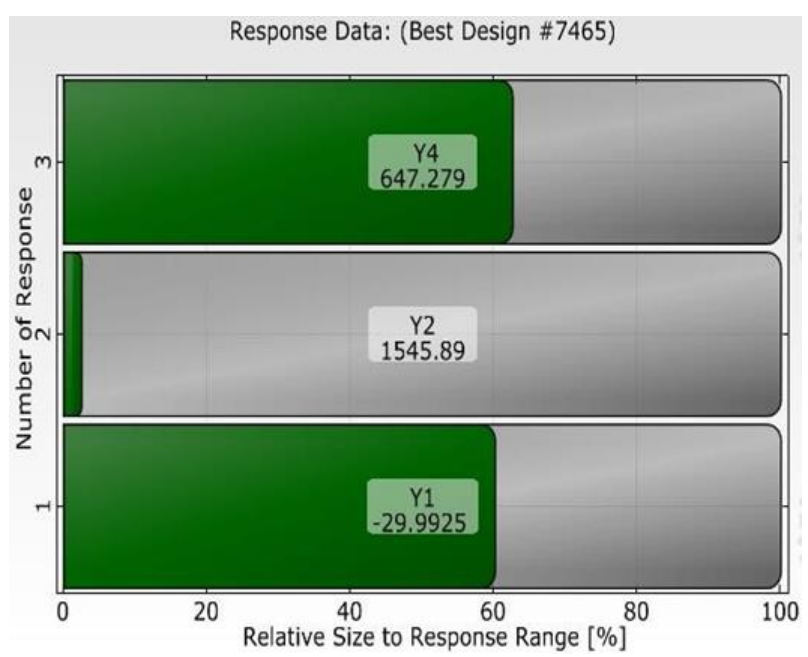

Fig. 28. Result of Optimized Design

After the optimization phase completed, the Finite element model was solved and verified according to optimum parameters. As seen on the following table, optimum values are so close to target values, So the optimization phase is successfully worked. Optimized dimensions are angle "20.5", Belleville diameter "50.612 mm", Belleville width "5.48 $\mathrm{mm}$ ", forming radius crossing point " $22.45 \mathrm{~mm}$ ", the thickness " $0.6 \mathrm{~mm}$ " and free height " $3.33 \mathrm{~mm}$ ". 
Table 4. Optimization comparison chart

\begin{tabular}{|c|c|c|c|c|c|}
\hline $\mid \begin{array}{l}\text { Variables } \\
(\mathbf{m m})\end{array}$ & X3 & X5 & $\mathrm{Y} 1(\mathrm{~N})$ & $\begin{array}{l}\text { Y2 } \\
\text { (MPa) }\end{array}$ & $\begin{array}{l}\mathrm{Y} 4 \\
(\mathrm{~N} / \mathrm{mm})\end{array}$ \\
\hline \begin{tabular}{|l|} 
Opt. \\
Evolution- \\
ary
\end{tabular} & 5.48 & 0.6 & 29.99 & 1545 & 647 \\
\hline FEA & & & 28.49 & 1544 & 621 \\
\hline $\begin{array}{l}\text { NLPQL } \\
\text { Min Stress }\end{array}$ & 5.48 & 0.6 & 29.99 & 1545 & 647 \\
\hline FEA & & & 28.49 & 1538 & 621 \\
\hline \begin{tabular}{|l|} 
Opt. \\
NLPQL \\
Max \\
Stiffness \\
\end{tabular} & & & 29.99 & 1545 & 647 \\
\hline FEA & & & -28.49 & 1544 & 621 \\
\hline
\end{tabular}

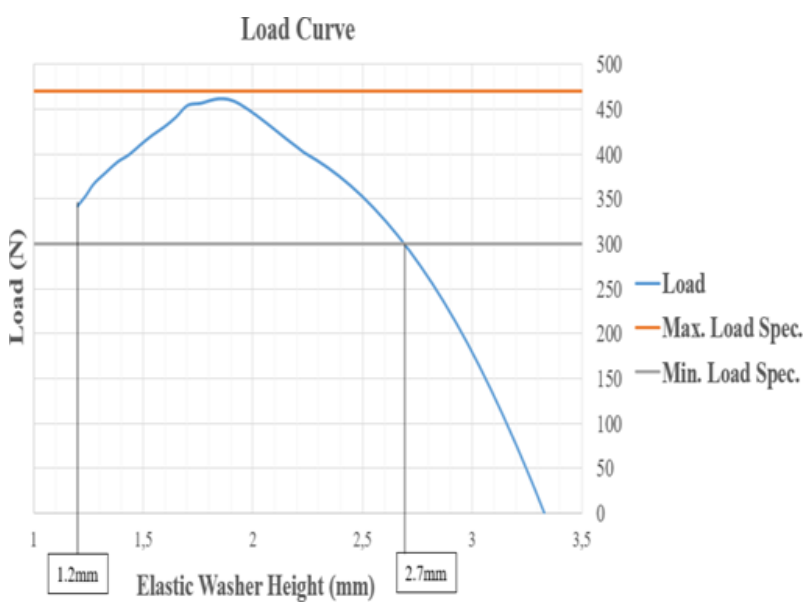

Fig. 29. Result of Load Curve for Optimized Design

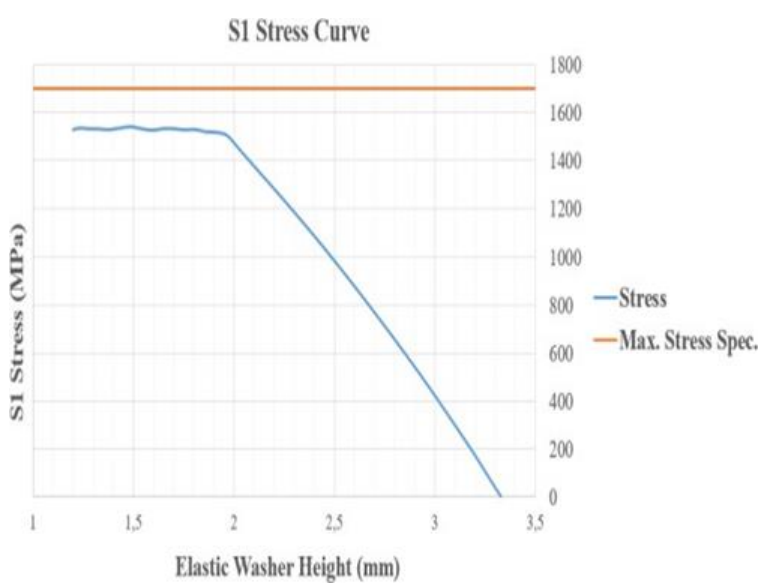

Fig. 30. Result of S1 Stress Curve for Optimized Design

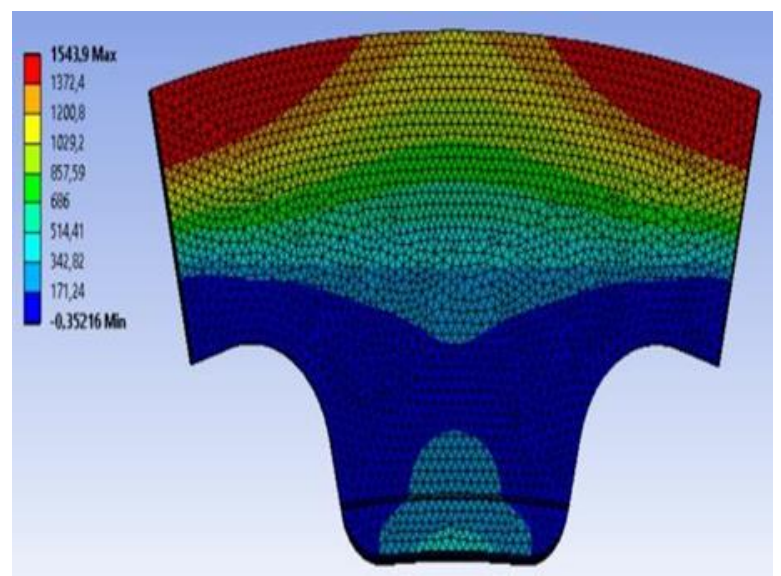

Fig.31. Result of S1 Stress Distribution for Optimized Design

\section{Conclusion}

There are five design variables, which are the angle, forming diameter, Belleville diameter, Belleville width and thickness, to define Belleville spring load and stress characteristic subjected to the defined inner and outer diameter of Belleville washer. However, angle and forming diameter need to be defined firstly since it defines free height which has a big impact on stress characteristic at the finite element calculation stage. After deciding angle and forming diameter, thickness, Belleville diameter and Belleville width dimensions are optimized so as to get optimum load and stress values. Thickness and Belleville diameter have so important for load characteristics of Belleville spring while forming diameter, angle and Belleville diameter have crucial importance on stress characteristic of Belleville washer. Belleville's diameter has an impact on the stress and load characteristic of the Belleville washer. Additionally, forming diameter is also so important on the stress characteristic of Belleville washer. Furthermore stiffness characteristic of Belleville spring depends on the Belleville dimension which impacts the effective working capacity of Belleville washer.

On the other hand, Optimization methods are not fully applied during the design phase in the automotive industry. Finite element analysis and optimization tools save development time and reduce costs. For this reason, more robust and creative designs must be created. The product development process can be done faster and more efficient by using CAE and optimization tools. In this study, the evolutionary algorithm and NLPQL were used to perform shape optimization into Optislang software while ANSYS workbench software was used for finite element calculations. 


\section{Nomenclature}

$\mathrm{N} \quad$ number of friction surface

RPM rotation of engine per minute

$\mathrm{T}$

X2 Belleville diameter ( $\mathrm{mm})$

X3 Belleville width (mm)

X5 Thickness (mm)

Y1 Load (N)

Y2 Principal Stress (Mpa)

Y4 Stiffness $(\mathrm{N} / \mathrm{mm})$

\section{References}

[1] Shree V.R.A., Veeraghavan S., Simhachalam B., Srinivas K., "Performance Comparison of Belleville Spring under Compressive Load using Experimental and Simulation Methods". International Conference on Emerging Trends in Materials and Manufacturing Engineering, 5 8486-8494, (2018).

[2] Weileun F, "Design of a Friction Clutch Using Dual Belleville Structures", ASME, 990 / Vol. 129, (2007).

[3] Patangtalo W., Aimmanee S., Chutima S. "A unified analysis of isotropic and composite Belleville springs", Thin Walled Structures, 109: 285-295, (2016).

[4] Dharan C.K.H., and Jesse A.B., "Composite disc springs", Composites, Part A 38; 2511-2516, (2007).

[5] Hwang, S. J., Chen, J. S., Liu, L., Ling, C. C., 'Modelling and Simulation of a powertrain system with automatic transmission" International Journal of Automobile Design, 2000, Vol. 23, No:1

[6] Macor, A., Benato, A., Rossetti, A., Bettio, Z., "Study and Simulation of a Hydraulic Hybrid Powertrain" $72^{\text {nd }}$ Conference of the Italian Thermal Machines Engineering Association, ATI2017, 6-8 September 2017, Lecce, Italy

[7] Genc, M. O., Kaya, N., "Modelling and Experimental Investigation of Clutch Damper Spring Stiffness on Truck Driving Comfort" International Journal of Advances on Automotive and Technology, Vol.2, No.2, pp.121-136, April 2018

[8] Wu, H., Wu, G., "Driveline Torsional Analysis and Clutch Damper Optimization for Reducing Gear Rattle" Shock and Vibration Volume 2016, Article ID 8434625 ,

[9] Chen, X., Wu, G., Wu., H., "The Nonlinear Characteristics Impact of Multi-Staged Stiffness Clutch Damper on the Automobile Creeping" SAE 2016 World Congress and Exhibition, DOI: 10.4271/2016-01-0431, April 2016.

[10] Kaya, N., 'Machining fixture locating and clampin
Computers in Industry, Vol. 57, pp. 112-120, 2006 